\title{
3D Modeling of Coronary Artery Bifurcations from CTA and Conventional Coronary Angiography
}

\author{
Rubén Cárdenes ${ }^{1}$, Jose Luis Díez ${ }^{2}$, Ignacio Larrabide ${ }^{1}$, \\ Hrvoje Bogunović ${ }^{1}$, and Alejandro F. Frangi ${ }^{1}$ \\ 1 Center for Computational Imaging \& Simulation Technologies in Biomedicine, \\ (CISTIB) - Universitat Pompeu Fabra and CIBER-BBN, Barcelona, Spain \\ ${ }^{2}$ Cardiology Department, Dr. Peset Hospital, Valencia, Spain
}

\begin{abstract}
Coronary artery bifurcations are regions where the atherosclerotic plaque appears more frequently and where the percutaneous treatment is more challenging. To analyze these important vascular regions, in this paper is proposed a method for the extraction of realistic $3 \mathrm{D}$ models of coronary bifurcations combining information from pre operative computer tomography angiography (CTA) to obtain the $3 \mathrm{D}$ structure of the vessels and pre and post operative conventional coronary angiography (CCA) to extract a more accurate estimation of the lumen radius before and after stenting. The method proposed is semiautomatic, starting from a set of user defined landmarks, and has been successfully applied to data from five patients that underwent endovascular treatment in a coronary bifurcation. The results obtained are satisfactory by visual inspection and in comparison with manual measurements.
\end{abstract}

\section{Introduction and Background}

Coronary artery bifurcations are places where atherosclerotic plaque formation occurs more frequently, and are challenging vascular regions for percutaneous treatment. Among the treatment techniques described for bifurcations, the one more appropriate for an individual case is still cause of controversy among endovascular interventionists. If a realistic 3D geometry of the bifurcation before and after treatment is available, a detailed analysis of the vascular morphology and its changes could respond to questions such as the level of success of the treatment employed or, in a large population study, what are the techniques more suitable for specific morphologies and locations. Also, computational fluid dynamics studies could be carried out using these geometries, and the study of the changes in hemodynamic parameters, could reveal regions of high risk of re-stenosis.

Several approaches have been proposed to obtain accurate segmentations of coronary arteries. Some important ones are minimum cost path techniques like the one presented in [1] that used a $4 \mathrm{D}$ representation of curves to obtain tubular structures from CT and MR data. Another similar approach was proposed in [2] 
to extract the vessel centerlines and then the vessel surface using fast marching. However, these and many other approaches are based on information about only one image modality, usually computer tomography angiography (CTA), and conventional coronary angiography (CCA) is not commonly used. In this paper we propose to combine these two modalities, taking advantage of each one to overcome the limitations of the other. Therefore, realistic 3D bifurcation geometries can be obtained using routine acquisition imaging modalities, with a semiautomatic method that provides the pre and post operative models of the vessels. This is, as far as we know, the first time that these two modalities are combined for reconstruction of coronary artery bifurcations.

\section{Method}

The method proposed here consists of three steps. First, the 3D structure of the bifurcation is obtained from CTA pre operative data using a ridge extraction method followed by a fast marching (FM) approach. After that, CCA images obtained before and after endovascular treatment are processed to obtain the vessel radii along the vessel centerlines, and finally, the models are constructed mapping the vessel radii to the $3 \mathrm{D}$ structure.

\subsection{D Centerline Extraction}

The first stage of the method uses the CTA image to extract the information of the vessel centerlines forming the bifurcation. In this step, the user specifies the center of the bifurcation in the CTA image, that will be denoted as $\boldsymbol{b}^{3 D}$, and a minimum of three points on the vessels forming the bifurcation, denoted as $\boldsymbol{p}_{1}^{3 D}$ for the proximal segment of the main artery, $\boldsymbol{p}_{2}^{3 D}$, for the distal part of the main artery and $\boldsymbol{p}_{3}^{3 D}$ for the secondary artery. Then, a ridge extraction is performed over the image using $\boldsymbol{p}_{1}^{3 D}, \boldsymbol{p}_{2}^{3 D}$, and $\boldsymbol{p}_{3}^{3 D}$ as seeds, to get the centerlines [3]. To obtain better results, the vessel enhancing filtering by [4] is applied to the CTA image prior to the ridge extraction.

The centerlines obtained from the ridge extraction process do not always form a connected set. Two segments are considered disconnected if they are separated by a distance greater than the step size used in the ridge extraction, (here the step size used is $0.3 \mathrm{~mm}$, lower than the minimum spatial resolution of the CTA, $0.445 \mathrm{~mm}$ ). These disconnections are due to several reasons, such as the relatively low spatial and temporal resolution of the CTA image, the noise, and especially due to lesions affecting the bifurcation. For this reason, a reconnection strategy is employed similarly to [2]. The method consists in finding for each disconnected segment, the closest point in any of the other segments, $\boldsymbol{y}$, using a distance map $u(\boldsymbol{x})$. Then, a minimum cost path is computed from $\boldsymbol{y}$ to the initial segment using $u(\boldsymbol{x})$. To compute $u(\boldsymbol{x})$ a FM algorithm is employed, solving the Eikonal equation:

$$
|\nabla u(\boldsymbol{x})| F(\boldsymbol{x})=1,
$$


and using the disconnected segment points as seeds. The FM computation is stopped when any of the other segment points, $\boldsymbol{y}$, is reached. Here, the speed function $F(\boldsymbol{x})$ has to be carefully selected. A constant speed function will provide an Euclidean distance map, that will connect the vessel centerlines by straight lines, thus, providing non realistic results (see green points in Fig. 1). In order to follow a more realistic trajectory of the vessels, we use a speed function that depends exponentially (as in [5]) on the local vessel structure as follows:

$$
F(\boldsymbol{x})=e^{(\alpha \cdot V(\boldsymbol{x}) / M)},
$$

where $V(\boldsymbol{x})$ is the multiscale vesselness of the CTA, computed as in [6], and $M$ is the maximum value in $V(\boldsymbol{x})$. Ten scales are used to compute $V(\boldsymbol{x})$, from 0.2 up to $4 \mathrm{~mm} . F(\boldsymbol{x})$ is designed to drive the FM process faster along vessels and the parameter $\alpha$ controls its strength. Low $\alpha$ values give a speed closer to constant everywhere, and high values increment the speed at high vesselness regions. Here we use $\alpha=2$ that provides good results in our experiments. The reconnection process is repeated for each segment until there is only one connected segment. The behavior of this reconnection strategy is shown in the phantom shown in Fig. 1] where three disconnected segments are connected following the vessel structures.

In Fig. 2 the centerline points obtained from the ridge extraction (red points) and reconnection step (green points) are shown for a left coronary artery tree. To take into account the calcified tissues of the plaque, that if present, are visible in the CTA, two different centerlines are computed for the pre and post operative models. For the post operative centerline, the ridge extraction is computed using the original CTA image. However, for the pre operative centerline, the calcifications are first subtracted from the CTA image using a threshold of 721 Hounsfield units [7, and then, the centerlines are computed again. Therefore, the pre operative centerlines deviate with respect to the previous ones at calcified lesions as shown in Fig. 2].
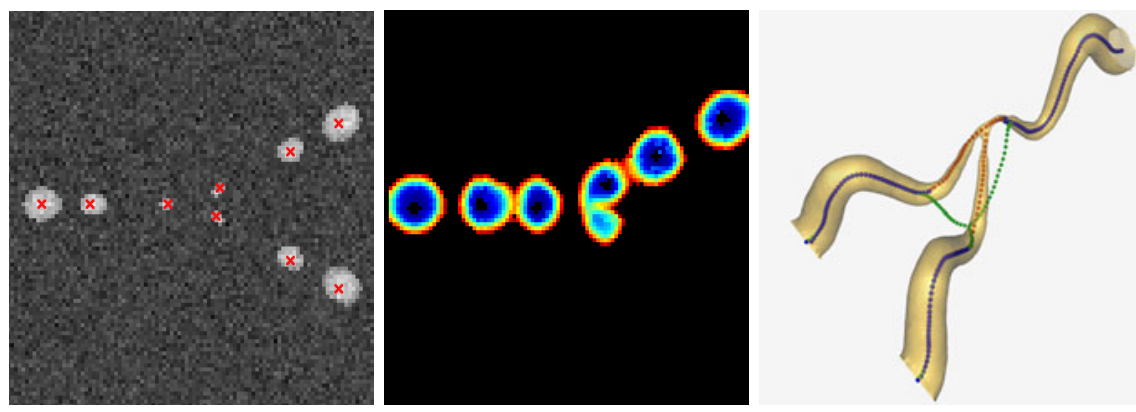

Fig. 1. Slice of a digital phantom with centerline points overlaid (left), same slice with $u(\boldsymbol{x})$ computed from one segment (middle), and 3D view of the phantom (right). Initial points are in blue, reconnected points in red, and in green connected points using $F(\boldsymbol{x})=1$. 


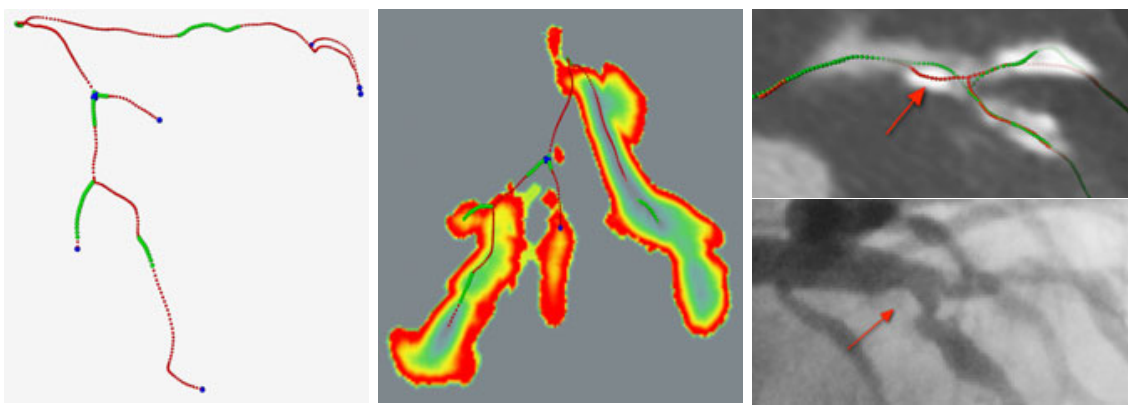

Fig. 2. Left: Initial centerline points (red) obtained from coronary CTA, connected centerline points using FM (green) and end points and bifurcation points (blue). Middle: slice of the distance field $u(\boldsymbol{x})$ from one segment. Right top: centerlines of the post operative model (red), and pre operative centerline (green) avoiding the calcified plaque pointed by arrows. Right bottom: corresponding CCA image.

\subsection{Processing of the 2D Angiographic Images}

In the following step, the vessel radii on the $\mathrm{CCA}$ images are calculated. In particular, two images are selected from pre and post operative views, using an angle where the area of the projection of the bifurcation is maximum. This projection corresponds approximately to the CCA image chosen by the clinician for an optimum visualization of the bifurcation, minimizing foreshortening and vessel overlap. Notice that, only vessel radii and lengths from the bifurcation center are used from CCA, and therefore there is no need to have a perfect projection matching between the CTA and the CCA. This is especially important because the vessel geometry extracted from CTA can vary with respect to the geometry extracted from CCA when the guide wire is inserted, that could change the vessels curvature (see Fig. 3). Therefore, our method does not need perfect
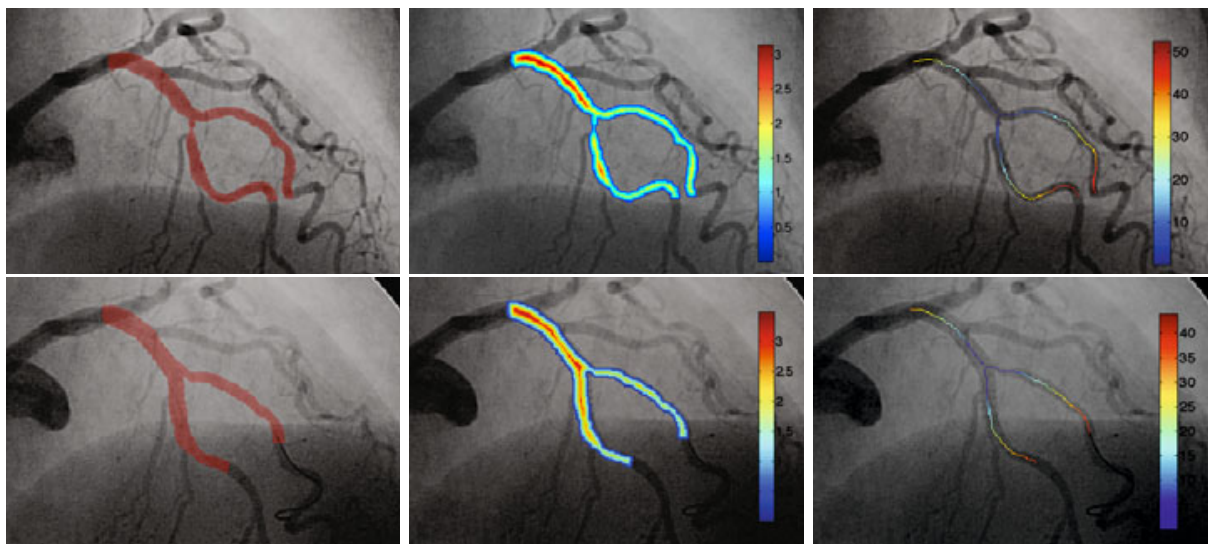

Fig. 3. CCA images. Top row: pre operative, bottom row: post operative. Overlay images are from left to right: segmentation, distances from the vessel boundary, and centerline colored with the lengths to $\boldsymbol{b}^{2 D}$. 
correspondence between CTA and CCA, being robust with respect to geometry changes and with respect to the cardiac phase used in both modalities, although it is chosen to be approximately the same. One alternative method could be to perform an elastic 2D registration between CCA and CTA projections to find radii correspondences, but the lack of information about the projection geometry of the CCA acquisition would increase considerably the complexity of the problem as well as the computational cost.

For each of the two projection images selected, the bifurcation center $\boldsymbol{b}^{2 D}$ is also manually selected, corresponding to the point $\boldsymbol{b}^{3 D}$. Also, three points on the branches are marked $\left(\boldsymbol{p}_{1}^{2 D}, \boldsymbol{p}_{2}^{2 D}\right.$ and $\left.\boldsymbol{p}_{3}^{2 D}\right)$, that will be used to assign correspondences between the 2D and $3 \mathrm{D}$ centerlines and to stop the $2 \mathrm{D}$ segmentation process, but that do not have to correspond to $\boldsymbol{p}_{i}^{3 D}$. The lumen in the CCA images are first segmented, then the vessel centerlines are computed, and finally the vessel radii are computed as the distances from the segmented boundary at the centerline points. For the segmentation, a level set approach is employed starting from $\boldsymbol{b}^{2 D}$ and is stopped when it reaches the user defined points $\boldsymbol{p}_{i}^{2 D}$. Two pre and post operative CCA images are shown in Fig. 3 with the segmentation, the distances from the segmented boundaries and the centerlines overlaid.

\subsection{Radius Mapping}

In the next step the radii values are mapped from $2 \mathrm{D}$ to $3 \mathrm{D}$, to construct the bifurcation models. Here, circular cross-section can be assumed for the arteries without losing too much accuracy in the majority of cases, because this assumption only fails in $20 \%$ to $30 \%$ of the lesions 8 .

The radius mapping is implemented using the centerline lengths. Let $\Gamma_{i}(s)$ and $\gamma_{i}(s)$ be the parameterized centerline curves of each vessel in $3 \mathrm{D}$ and $2 \mathrm{D}$ respectively, where $\Gamma_{i}(0)=\boldsymbol{b}^{3 D}, \Gamma_{i}(1)=\boldsymbol{p}_{i}^{3 D}$ and $\gamma_{i}(0)=\boldsymbol{b}^{2 D}$. Then, we choose $\gamma_{i}(1)$ as the $2 \mathrm{D}$ point such as corresponding curves have the same length, $L\left(\Gamma_{i}(1)\right)=L\left(\gamma_{i}(1)\right)$, where the length function $L(\gamma(s))$ is given by

$$
L(\gamma(s))=\int_{0}^{s}|\dot{\gamma}(t)| \gamma(t) d t .
$$

Then, a radius value is assigned to each point of $\Gamma_{i}$, using the radii values computed previously in the CCA images. The mapping is performed using distances from the bifurcation center, such that if $R_{i}(d)$ and $r_{i}(d)$, are the radii in $3 \mathrm{D}$ and 2D at distance $d$ from $\boldsymbol{b}^{3 D}$ and $\boldsymbol{b}^{2 D}$ respectively, at the $i^{\text {th }}$ branch, then, the radius mapping can be expressed as $R_{i}\left(L\left(\Gamma_{i}(s)\right)\right)=r_{i}\left(L\left(\gamma_{i}(s)\right)\right), \forall s \in[0,1]$.

In general, there is no exact correspondence between the points $\Gamma_{i}(s)$ and $\gamma_{i}(s)$ due to foreshortening. However, we can assume that in a small region near $\boldsymbol{b}^{3 D}$, the vessel centerlines lay near a plane [9], and thus, the foreshortening effect is minimum on this plane and the radius mapping can be applied. Finally, the obtained surfaces are smoothed to avoid sharp transitions at vessel junctions. 


\section{Results}

We have selected 5 patients for this study, affected by occlusion in a coronary bifurcation and eligible for percutaneous intervention. All the patients had a pre operative CTA scan, which was acquired in a 64 multi-slice scanner from GE Medical Systems, with an image resolution of $0.445 \times 0.445 \times 0.625 \mathrm{~mm}$, and at least two sequences of pre and post operative CCAs as described above.

In order to show our results, we have selected for each case, the CTA oblique slice that approximates best to the plane formed locally by the bifurcation vessels, that also fits best with the CCAs used, and will be denoted as bifurcation plane. This plane is shown in Fig. 5 (left) for one case, that corresponds to the tangent plane of the epicardium. Fig. 4 illustrates the bifurcation region on each CCA (top row), the CTA bifurcation plane with the models and a manual segmentation outlines overlaid (middle row), and the pre and post operative bifurcation models for the five patients (bottom row). The differences between the two models and the matching with the real images are clearly seen. In the first and fifth cases, the secondary branch is bigger in the pre operative model (green color inside), which is in accordance with the diameters observed in the CCAs, and could be due to partial occlusion of the secondary branch after stenting.
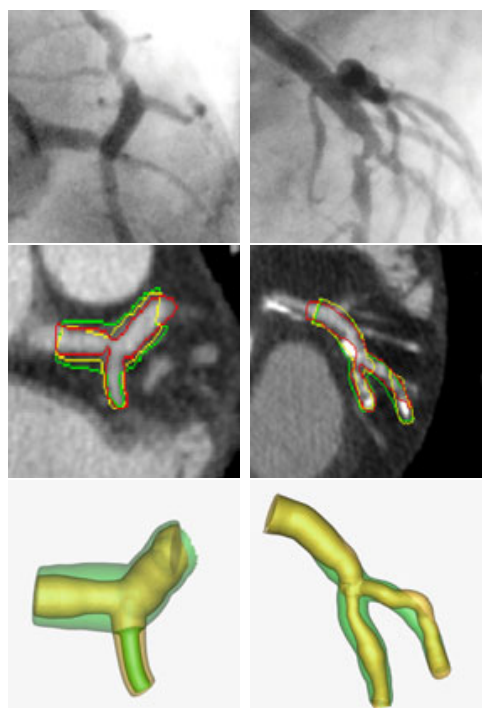
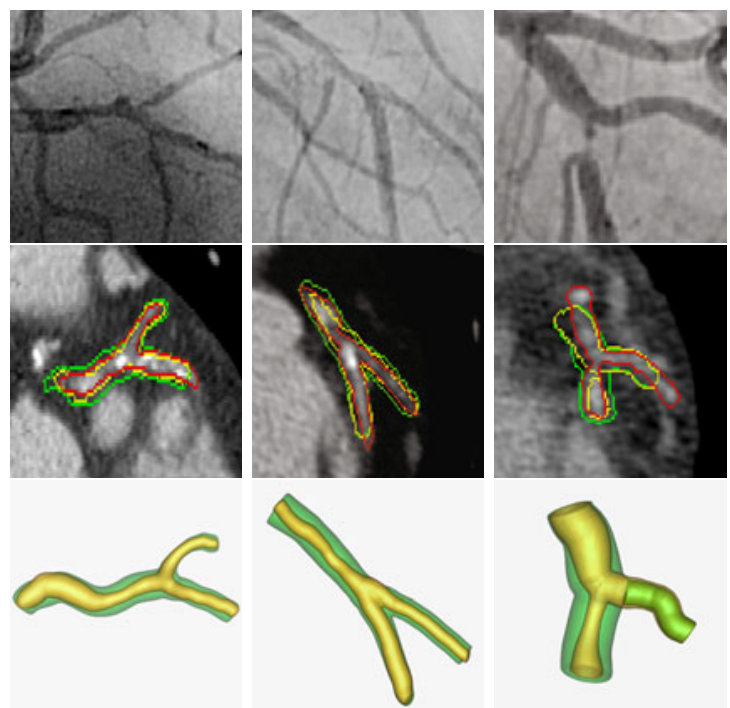

Fig. 4. Detail of the CCAs used (top row), corresponding bifurcation plane from the CTA with manual segmentation (red), pre (yellow) and post operative (green) outlines overlaid (middle row), and 3D bifurcation models (bottom row); in yellow and green the pre and post operative models respectively. 

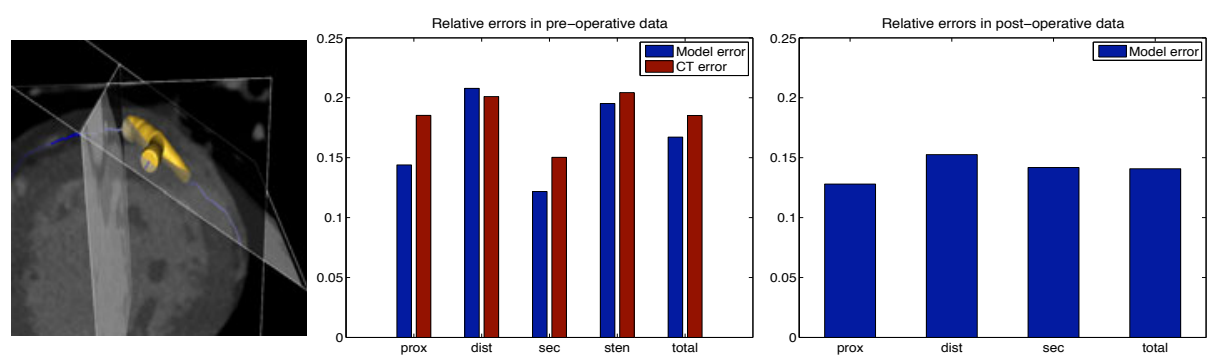

Fig. 5. Bifurcation plane in one case (left), and relative errors obtained in the diameters measures for the pre operative data (middle) and for the post operative data (right)

\section{Comparison with Manual Measurements}

To evaluate our results, two comparisons have been carried out. First, manual segmentation is performed in the bifurcation plane extracted from the CTA, and is compared with the pre operative model at the same plane, computing the dice similarity index between the two regions, whose boundaries are shown in Fig. 4 (middle row). The values obtained are shown in table 1, where a good agreement is found (0.7675 in average).

A second comparison has been done measuring the three vessels diameters in the CTA, the CCAs and in our models using an annotation tool. The diameter at the stenosis is also measured in all the pre operative data. Then, considering the diameters from the $\mathrm{CCA}$ as the ground truth, the relative errors of our method and from CTA are computed for the pre operative data, see Fig. 5 (middle). These errors are lower in our models than in CTA, showing the importance of including the radius information from CCA. The errors obtained between our post operative models and the post operative CCAs are also plotted in Fig. 5 (right), where the values obtained are slightly smaller than before.

Table 1. Dice similarity index computed between manual delineations in the bifurcation plane and a slice of the same plane of the pre operative models for the 5 patients.

\begin{tabular}{lccccc|c}
\hline & patient 1 & patient 2 & patient 3 & patient 4 & patient 5 & average \\
\hline DS : & 0.8171 & 0.7217 & 0.8244 & 0.8197 & 0.6545 & 0.7675 \\
\hline
\end{tabular}

\section{Conclusions}

We have proposed a method for realistic modeling of coronary bifurcations combining information from two modalities: CTA and CCA. We have shown the advantages of using both modalities together: from CTA, the 3D vessel trajectories and the visible atherosclerotic plaque are used, and from CCA we obtain a more accurate estimation of the lumen radius, before and after stenting. The results presented from five patients are successful, and the models obtained match 
well with both the CTA and the CCA data as shown above. Numerical comparison with manual segmentation shows a good accuracy. Also, from comparisons of the diameters we have also shown that this method can improve the accuracy with respect to CTA used alone. Therefore, using images acquired during normal clinical practice, it is possible to obtain accurate models from specific regions of the coronary artery tree, before and after percutaneous intervention. These accurate models can be extremely useful to assess geometrically the outcome of endovascular interventions and they can be obtained very efficiently.

Two assumptions have been used: circular cross section of the vessels, that is not true only in $20 \%$ to $30 \%$ of the lesions, and planar correspondence between the CCAs and the bifurcation vessels in a small region around its center. From the results obtained, it is shown qualitatively and quantitatively, that both approximations are valid with the data sets used here, and therefore this method shows to be a good technique to obtain realistic results. However, a wider study with a larger population, analyzing user input variability, cardiac phase selection and acquisition plane selection, is still needed to fully validate this method.

Acknowledgements. This work has been funded by a CDTI CENIT-cvREMOD grant of the Spanish Ministry of Science and Innovation, R.C. is funded by a Beatriu de Pinós fellowship from AGAUR, and A.F.F. is partially funded by the ICREAAcademia programme, from the Regional Government of Catalonia.

\section{References}

1. Li, H., Yezzi, A.: Vessels as 4-D curves: Global minimal 4-D paths to extract 3-D tubular surfaces and centerlines. IEEE T. Med. Imag. 26(9), 1213-1223 (2007)

2. Mueller, D., Maeder, A.: Robust semi-automated path extraction for visualising stenosis of the coronary arteries. Comput. Med. Imag. Grap. 32, 463-475 (2008)

3. Aylward, S., Pace, D., et al.: TubeTK, segmentation, registration, and analysis of tubular structures in images. Kitware, Inc. (2010)

4. Manniesing, R., Viergever, M.A., Niessen, W.J.: Vessel enhancing diffusion: A scale space representation of vessel structures. Med. Image Anal. 10(6), 815-825 (2006)

5. Hassouna, M.S., Farag, A.A.: Variational curve skeletons using gradient vector flow. IEEE T. Pattern Anal. 31(12), 2257-2274 (2009)

6. Frangi, A.F., Niessen, W.J., Vincken, K.L., Viergever, M.A.: Multiscale vessel enhancement filtering. In: Wells, W.M., Colchester, A., Delp, S.L. (eds.) MICCAI 1998. LNCS, vol. 1496, pp. 130-137. Springer, Heidelberg (1998)

7. Sakakura, K., Yasu, T., Kobayashi, Y., Katayama, T., Sugawara, Y., Funayama, H., Takagi, Y., Ikeda, N., Ishida, T., Tsuruya, Y., Kubo, N., Saito, M.: Noninvasive tissue characterization of coronary arterial plaque by 16-slice CT in acute coronary syndrome. Angiology 52(2), 155-160 (2006)

8. Arbab-Zadeh, A., Texter, J., Ostbye, K.: Quantification of lumen stenoses with known dimensions by conventional angiography and CT? implications for the use of angiography as a gold standard. Heart 96, 1358-1363 (2010)

9. Fung, Y.: Biomechanics, 2nd edn. Springer, New York (1997) 